\title{
A Jumpstart for Biochemistry
}

\author{
RNA can replicate in conditions that could be found on the early Earth, \\ suggesting a possible step in the origin of life.
}

\author{
By Michael Schirber
}

( ne theory for the origin of life proposes that the initial biochemical steps were taken by RNA, a molecular cousin to DNA. Reactions involving RNA alone can create copies of short RNA strands, and a new study shows that this replication can occur in a lab setting that mimics a small pore in a volcanically heated rock-a presumably common setting on early Earth. Researchers placed RNA in a small chamber with hot and cold regions and observed molecular movement that helped drive multiple cycles of RNA replication. The number of RNA strands increased by as much as 200,000 times, suggesting that this RNA chemistry may have been a stepping stone to building more complex biomolecules in the prebiotic Earth environment.

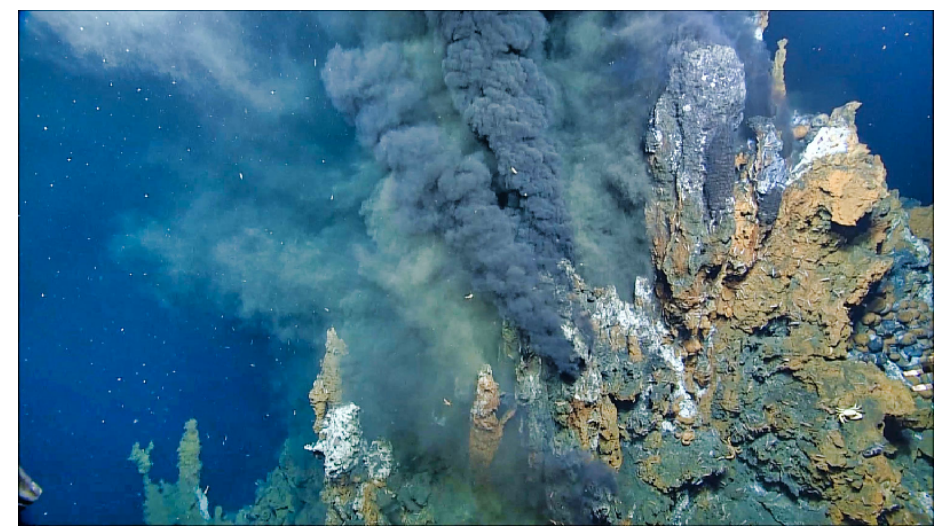

Where life began? Hydrothermal vents like this one in the southwest Pacific Ocean could have hosted the earliest biochemical events that led to the start of life on Earth. Experiments now show that RNA can replicate on its own in conditions that may have prevailed within these rocks. Credit: Schmidt Ocean Inst./ROV ROPOS
In present-day organisms, the two main biochemical functions for life-storing information and driving chemical reactions-are carried out, respectively, by DNA and proteins. However, it seems improbable that both of these complicated types of molecules evolved simultaneously. So biologists have proposed that RNA performed both roles to jumpstart life on Earth [1]. Like DNA, RNA is composed from a set of four nucleotides that can be arranged to form a genetic code. RNA can also assume structural forms that act as catalysts that drive reactions. One problem for origin-of-life theories is that these RNA catalysts, or "ribozymes," are highly temperature sensitive, raising doubts about their effectiveness in a natural setting, says Dieter Braun from the Ludwig Maximilian University of Munich (LMU).

Braun and his colleagues have now demonstrated an RNA-based replication cycle within an environment that could have existed in the early Earth. "We actually bring these molecules into the wild, so to speak," Braun says. The cycle's chemical engine is a recently discovered ribozyme that can make a copy of a short strand of RNA called the template [2]. In previous studies by Braun's collaborators, the ribozyme replicated various templates in experiments where the temperature was briefly ramped up from $20^{\circ}$ to $60^{\circ}$ Celsius every half hour or so. This high-temperature spike was needed to force the RNA copy to separate from the template, so that the process could be repeated.

On Earth's surface, temperatures don't usually spike quickly in time, but there are geological settings where the temperature varies abruptly in space. Braun and colleagues tested whether such a temperature gradient could drive RNA replication. The team placed a solution of ribozymes, templates, and an assortment of RNA pieces in a 5-mm-diameter, disk-shaped 
chamber. They created a steep temperature gradient by heating the center of the chamber with a laser. The temperature variations induced two types of flows: convection and thermophoresis. Convection is a looping movement of fluid caused by warmer liquid rising and colder liquid sinking. Thermophoresis is the motion of molecules along a temperature gradient-typically from hot to cold.

For RNA, longer molecules are more affected by thermophoresis than shorter ones, explains team member Annalena Salditt from LMU. As such, the 200-nucleotide-long ribozyme is pushed away from the hottest region, protecting it from heat degradation. "The ribozyme sits in the cold and waits for other RNA molecules," explains Braun. When a template molecule floats by, the ribozyme grabs it and builds up a copy using other small RNA pieces in the fluid. Once the replication is done, the template and its copy are pushed by convection into the hot region, where they separate. Afterwards, the template and its copy move back to the cold, where each can be replicated. This doubling of the RNA molecules in each cycle leads to exponential growth, as evidenced by as much as a 200,000-fold increase in the number of template RNA molecules in the chamber after a 24-hour period.

The ribozyme can only copy templates that are at most 30 nucleotides long, which means it can't replicate itself. Such self-replication is seen as a key requirement for prebiotic chemistry. Still, Braun believes that self-replication may not be too far off, if researchers can identify a complementary ribozyme that glues together different RNA strands.

Thomas Cech, a biochemist from the University of Colorado, Boulder, sees important progress. "RNA self-replication experiments are typically done under controlled laboratory conditions, where all of the molecular ingredients are at equilibrium, homogeneous, and well-mixed," he says. The researchers have performed RNA chemistry in a more variable environment that is likely closer to the origin-of-life conditions. "As the environment is made arguably more realistic, RNA self-replication becomes more plausible," Cech says.

This research is published in Physical Review Letters.

Michael Schirber is a Corresponding Editor for Physics based in Lyon, France.

\section{REFERENCES}

1. W. Gilbert, "Origin of life: The RNA world," Nature 319, 618 (1986).

2. D. P. Horning and G. F. Joyce, "Amplification of RNA by an RNA polymerase ribozyme," Proc. Natl. Acad. Sci. U.S.A. 113, 9786 (2016). 